\title{
Spectral Modeling of Switched-Mode Power Converters in Discontinuous Conduction Mode
}

\author{
K.T. Chau, C.F. Lam ${ }^{\dagger}$ and C.C. Chan ${ }^{*}$ \\ * The University of Hong Kong, Hong Kong \\ ${ }^{\dagger}$ Tuen Mun Technical Institute, Hong Kong
}

\begin{abstract}
Analytical spectral modeling of switched-mode PWM converters, operating in the discontinuous conduction mode, is presented. The modeling approach is to derive analytical expressions of the converter output response using the extended Volterra functional series. Nonlinear frequency responses, including additional DC offset as well as significant subharmonic and higher harmonic components, due to large-signal perturbations of both the duty ratio and source voltage, are investigated. Their vulnerability to switching frequencies are also described. This modeling approach has been applied to various PWM converters, boost, buck and buck-boost types, operating in the discontinuous conduction mode, and the results are verified.
\end{abstract}

\section{INTRODUCTION}

Due to the switching nature of power converters, their dynamical behavior is inherently nonlinear. Although a number of small-signal modeling techniques have been developed to assess their small-signal frequency responses and dynamical stability, these techniques can neither handle largesignal frequency responses nor predict spectral contamination. Moreover, linear system theory such as superposition theorem can no longer be used for multiple independent inputs with large-signal perturbations.

In 1991, the classical Volterra functional series was firstly applied to model the control-to-output response of switchedmode boost PWM converters [1]. Instead of being confined to single-input single-output converters, the Volterra functional series was extended in a new way and applied to the nonlinear modeling of switched-mode boost PWM converters with multiple independent inputs and the spectral modeling of various switched-mode PWM converters [2]. However, these papers have made the same assumption that the converter must operate in the continuous conduction mode. In this mode of operation, the inductor current is always positive and only two topological stages exist. Switching between stages depends only on the externally-controlled duty ratio. Once the continuous conduction mode can be maintained by adopting a sufficiently high switching frequency or large inductance, the switching frequency has no influence on the converter dynamical performance.

Either due to design or because of light load, the converter can operate in the discontinuous conduction mode. In this mode of operation, there are three topological stages in which the additional stage is due to the fact that the inductor current becomes zero before the end of switching period. Thus, there is a problem that switching between stages not only depends on the externally-controlled duty ratio but also on the internal circuit parameters.

It is the purpose of this paper to further extend the application of Volterra functional series to the spectral modeling of switched-mode power converters operating in the discontinuous conduction mode. Analytical spectral models of various PWM converters are derived.

\section{REVIEW}

The derivation of Volterra transfer functions for nonlinear systems with multiple independent inputs in [2] is applicable to this paper because it is independent of the modes of operation. Without duplicating the corresponding derivation while maintaining the readability, the Volterra model of switchedmode PWM converters is shown in Fig. 1. In this model, superscripts $a$ and $b$ represent input perturbations of the duty ratio and source voltage, respectively, $H_{1}^{a}$ and $H_{1}^{b}$ are the 1st-order Volterra transfer functions, $\mathrm{H}_{2}^{a a}, \mathrm{H}_{2}^{b b}$ and $\mathrm{H}_{2}^{a b}$ are the 2nd-order transfer functions, and $H_{3}^{a a a}, H_{3}^{b b b}, H_{3}^{a a b}$ and $H_{3}^{a b b}$ are the 3rd-order functions. $H_{1}^{a}, H_{1}^{b}, H_{2}^{a a}, H_{2}^{b b}$, $H_{3}^{a a a}$ and $H_{3}^{b b b}$ are for describing the individual contribution of each input to the output response, while $H_{2}^{a b}, H_{3}^{a a b}$ and $H_{3}^{a b b}$ are for the interaction of two inputs to affect the output response. In general, the first three orders are sufficient to represent the output response. The higher-order transfer functions can similarly be derived with ever increasing tedium.

Also, the derivation of spectral frequency responses of switched-mode PWM converters in [2] is applicable to this paper. Although the detailed derivation is omitted, the general expressions of the 1st- and 2nd-order responses are listed in Table I, where $A_{i}$ and $B_{i}$ are complex amplitudes of the $i$ th tone of $a$ and $b$, respectively, $A_{i}^{*}$ and $B_{i}^{*}$ are their conjugates, and $A_{i}^{(*)}$ and $B_{i}^{\left({ }^{*}\right)}$ are their optional conjugates corresponding to the minus sign of $\pm s_{a i}$ and $\pm s_{b i}$, respectively. It should be noted that when a negative frequency is encountered, the complex conjugation of its amplitude becomes a realistic amplitude because of the general properties $H_{n}\left(-s_{1}, \cdots,-s_{n}\right)=H_{n}^{*}\left(s_{1}, \cdots, s_{n}\right)$. 


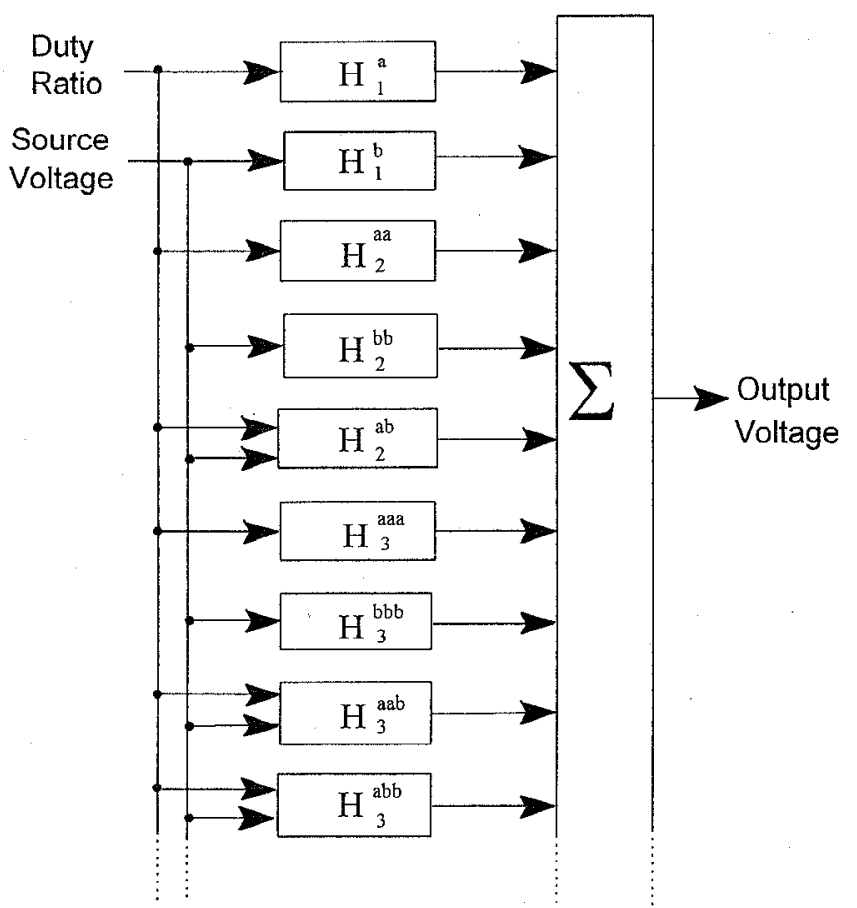

Fig. 1 Volterra model of switched-mode PWM converters

TABLE I

THE 1ST-AND 2ND-ORDER RESPONSES

\begin{tabular}{ll}
\hline \multicolumn{1}{c}{ Frequency } & \multicolumn{1}{c}{ Amplitude } \\
\hline$s_{a i}(i=1,2)$ & $A_{i} H_{1}^{a}\left(s_{a i}\right)$ \\
$s_{b i}(i=1,2)$ & $B_{i} H_{1}^{b}\left(s_{b i}\right)$ \\
$s_{a i} \pm s_{a i}(i=1,2)$ & $A_{i} A_{i}^{(*)} H_{2}^{a a}\left(s_{a i}, \pm s_{a i}\right)$ \\
$s_{b i} \pm s_{b i}(i=1,2)$ & $B_{i} B_{i}^{(*)} H_{2}^{b b}\left(s_{b i}, \pm s_{b i}\right)$ \\
$s_{a i} \pm s_{a j}(i, j=1,2 ; i<j)$ & $A_{i} A_{j}^{(*)} H_{2}^{a a}\left(s_{a i}, \pm s_{a j}\right)$ \\
$s_{b i} \pm s_{b j}(i, j=1,2 ; i<j)$ & $B_{i} B_{j}^{(*)} H_{2}^{b b}\left(s_{b i}, \pm s_{b j}\right)$ \\
$s_{a i} \pm s_{b j}(i, j=1,2)$ & $0.5 A_{i} B_{j}^{(*)} H_{2}^{a b}\left(s_{a i}, \pm s_{b j}\right)$ \\
\hline
\end{tabular}

\section{SPECTRAL MODELING}

Since the PWM boost, buck and buck-boost converters are the basic converters from which the other converters are derived, the spectral modeling approach is exemplified using these basic converters. As shown in Fig. 2, these converters consist of the same circuit elements: power switch $S$, power diode $D$, inductor $L$, capacitor $C$ and resistor $R$. All parasitics such as equivalent series resistances, junction capacitances and stray inductances are neglected to simplify the derivation and explanation. In fact, these parasitics can be included with increasing complexity. The input excitations are the duty ratio $d_{1}$ and source voltage $v_{g}$, while the output response is the output voltage $v_{o}$.
Fig. 3 shows a general inductor current $i_{L}$ waveform of these converters operating in the discontinuous conduction mode. Each switching period $T_{s}$ is divided into three stages: the 1st stage corresponds with $S$ on and $D$ off, the 2nd stage with $S$ off and $D$ on, and the 3rd stage with $S$ off and $D$ off. Their intervals are denoted as $d_{1} T_{s}, d_{2} T_{s}$ and $d_{3} T_{s}$, respectively [3].

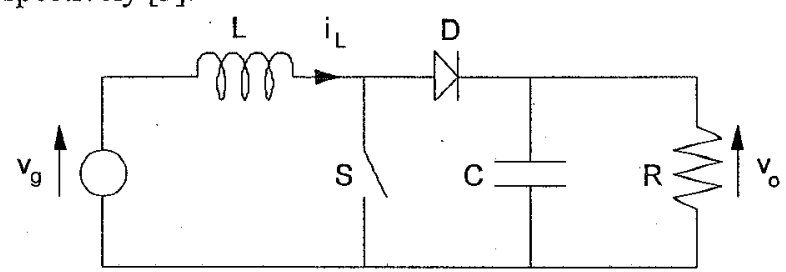

(a)

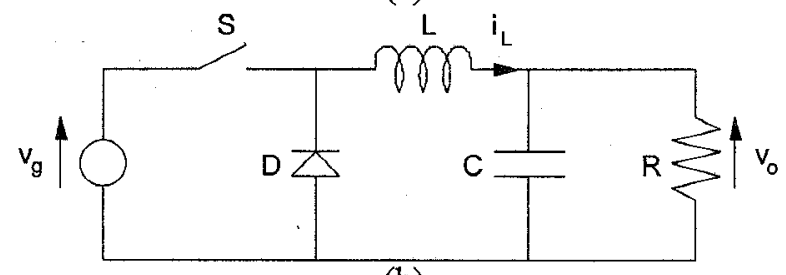

(b)

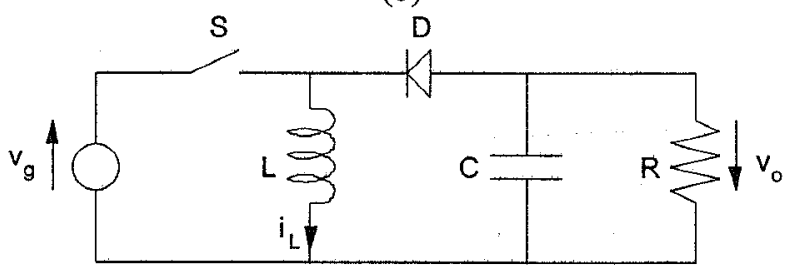

(c)

Fig. 2 PWM converters: (a) boost (b) buck (c) buck-boost

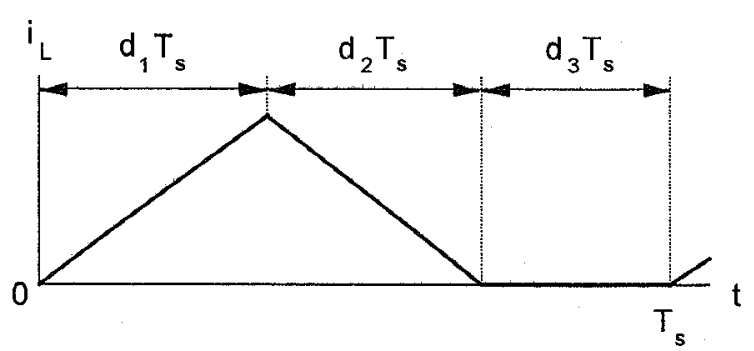

Fig. 3 Inductor current in discontinuous conduction mode

\section{A. Boost Converter}

Firstly, a continuous-time large-signal model describing the converter dynamical behaviour is formulated using the wellknown state-space averaging technique. This technique is valid when the converter natural frequencies are all well below its switching frequency, which is the case for a practical PWM converter with switching frequencies ranging from tens of kilohertz to hundreds of kilohertz. The resulting time-invariant model is given by

$$
\dot{\mathbf{x}}=\left(d_{1} \mathbf{C}_{1}+d_{2} \mathbf{C}_{2}+d_{3} \mathbf{C}_{3}\right) \mathbf{x}+\left(d_{1} \mathbf{D}_{1}+d_{2} \mathbf{D}_{2}+d_{3} \mathbf{D}_{3}\right) v_{g}
$$


where $\mathbf{x}=\left[\begin{array}{ll}i_{L} & v_{o}\end{array}\right]^{\mathrm{T}}$ is the state vector, and $\mathbf{C}_{1}, \mathbf{C}_{2}, \mathbf{C}_{3}$, $\mathbf{D}_{1}, \mathbf{D}_{2}$ and $\mathbf{D}_{3}$ are given by

$$
\begin{array}{ll}
\mathbf{C}_{1}=\left[\begin{array}{cc}
0 & 0 \\
0 & -\frac{1}{C R}
\end{array}\right] \quad \mathbf{C}_{2}=\left[\begin{array}{cc}
0 & -\frac{1}{L} \\
\frac{1}{C} & -\frac{1}{C R}
\end{array}\right] \quad \mathbf{C}_{3}=\left[\begin{array}{cc}
0 & 0 \\
0 & -\frac{1}{C R}
\end{array}\right] \\
\mathbf{D}_{1}=\left[\begin{array}{ll}
\frac{1}{L} & 0
\end{array}\right]^{\mathrm{T}} & \mathbf{D}_{2}=\left[\begin{array}{ll}
\frac{1}{L} & 0
\end{array}\right]^{\mathrm{T}} \quad \mathbf{D}_{3}=\left[\begin{array}{ll}
0 & 0
\end{array}\right]^{\mathrm{T}}
\end{array}
$$

In order to express $v_{o}$ in terms of $d_{1}$ and $v_{g}$ analytically, those dependent variables $d_{2}$ and $d_{3}$ have to be rewritten. Since the summation of intervals $d_{1} T_{s}, d_{2} T_{s}$ and $d_{3} T_{s}$ must equal $T_{s}$, this relationship can be expressed as

$d_{1}+d_{2}+d_{3}=1$

Since $i_{L}$ does not have free boundary conditions, but fixed at zero, a constraint can be expressed as

$\dot{i}_{L}=0$

which follows that $i_{L}$ ceases to be a true state-space variable. Since a perturbation of the instantaneous $i_{L}$ causes a perturbation of the average $i_{L}$, the average $i_{L}$ is the quantity which reflects the effect of the introduced perturbation. Thus, a second constraint can be obtained from the converter circuit itself and is given by

$i_{L}=\frac{v_{\mathrm{g}}}{2 L} d_{1} T_{s}$

By substituting (2)-(5) into (1), the desired large-signal dynamical equation describing the converter output voltage response can be obtained as

$\dot{v}_{o}=\frac{d_{1}^{2} v_{g}^{2} T_{s}}{2 C L\left(v_{o}-v_{g}\right)}-\frac{v_{o}}{C R}$

Perturbations in the input excitations $d_{1}$ and $v_{g}$ cause a perturbation in the output response $v_{o}$. Thus

$$
\begin{aligned}
& d_{1}=\bar{d}_{1}+\widetilde{d}_{1} \\
& v_{g}=\bar{v}_{g}+\widetilde{v}_{g} \\
& v_{o}=\bar{v}_{o}+\widetilde{v}_{o}
\end{aligned}
$$

where the steady-state and perturbed quantities are indicated with a bar and a tilde, respectively. By substituting (7) into (6), and using the fact that $\dot{\bar{v}}_{o}$ is always zero, (6) can be rewritten as

$$
\dot{\widetilde{v}}_{o}=\frac{\left(\bar{d}_{1}+\tilde{d}_{1}\right)^{2}\left(\bar{v}_{g}+\widetilde{v}_{g}\right)^{2} T_{s}}{2 C L\left(\bar{v}_{o}+\widetilde{v}_{o}-\bar{v}_{g}-\widetilde{v}_{g}\right)}-\frac{\bar{v}_{o}+\widetilde{v}_{o}}{C R}
$$

By equating the steady-state quantities in (8), the steady-state output voltage can be obtained as

$\bar{v}_{o}=\frac{\bar{v}_{g}}{2}\left(1+\sqrt{1+2 \tau \bar{d}_{I}^{2}}\right)$ where $\tau=T_{s} R / L$ is the dimensionless ratio of switching period to time constant.

Having obtained the dynamical equation of the converter output voltage, the $n$ th-order Volterra transfer functions can be determined by using the probing method [4]. In order to determine the first three Volterra transfer functions, the input excitations are represented by three-exponential expressions

$$
\begin{aligned}
& \widetilde{d}_{1}=a(t)=\sum_{i=1}^{3} \exp \left(s_{a i} t\right) \\
& \widetilde{v}_{g}=b(t)=\sum_{i=1}^{3} \exp \left(s_{b i} t\right)
\end{aligned}
$$

and the resulting output response is represented by

$$
\begin{aligned}
\widetilde{v}_{o}=y( & t)=\sum_{i=1}^{3} M_{1}^{a}\left(s_{a i}\right) \exp \left(s_{a i} t\right)+\sum_{i=1}^{3} M_{1}^{a}\left(s_{b i}\right) \exp \left(s_{b i} t\right) \\
& +\sum_{i=1}^{3} \sum_{j=i}^{3} M_{2}^{a a}\left(s_{a i}, s_{a j}\right) \exp \left(s_{a i} t+s_{a j} t\right) \\
& +\sum_{i=1}^{3} \sum_{j=i}^{3} M_{2}^{b b}\left(s_{b i}, s_{b j}\right) \exp \left(s_{b i} t+s_{b j} t\right) \\
& +\sum_{i=1}^{3} \sum_{j=1}^{3} M_{2}^{a b}\left(s_{a i}, s_{b j}\right) \exp \left(s_{a i} t+s_{b j} t\right) \\
& +\sum_{i=1}^{3} \sum_{j=i}^{3} \sum_{k=j}^{3} M_{3}^{a a a}\left(s_{a i}, s_{a j}, s_{a k}\right) \exp \left(s_{a i} t+s_{a j} t+s_{a k} t\right) \\
& +\sum_{i=1}^{3} \sum_{j=i}^{3} \sum_{k=j}^{3} M_{3}^{b b b}\left(s_{b i}, s_{b j}, s_{b k}\right) \exp \left(s_{b i} t+s_{b j} t+s_{b k} t\right) \\
& +\sum_{i=1}^{3} \sum_{j=i}^{3} \sum_{k=1}^{3} M_{3}^{a a b}\left(s_{a i}, s_{a j}, s_{b k}\right) \exp \left(s_{a i} t+s_{a j} t+s_{b k} t\right) \\
& +\sum_{i=1}^{3} \sum_{j=1}^{3} \sum_{k=j}^{3} M_{3}^{a b b}\left(s_{a i}, s_{b j}, s_{b k}\right) \exp \left(s_{a i} t+s_{b j} t+s_{b k} t\right)
\end{aligned}
$$

By substituting (10) and (11) into (8), and equating relevant exponentials, the corresponding coefficients can be obtained as $M_{1}^{a}(s)=\tau \bar{d}_{1} \bar{v}_{g}^{2} / D_{1}(s)$

$M_{1}^{b}(s)=\left(\bar{v}_{o}+\tau \bar{d}_{1}^{2} \bar{v}_{g}\right) / D_{1}(s)$

$D_{1}(s)=\left(2 \bar{v}_{o}-\bar{v}_{g}\right)+C R\left(\bar{v}_{o}-\bar{v}_{g}\right) s$

$M_{2}^{a a}\left(s_{1}, s_{2}\right)=\left(\tau \bar{v}_{g}^{2}-\cdots\right) / D_{2}\left(s_{1}, s_{2}\right)$

$M_{2}^{b b}\left(s_{1}, s_{2}\right)=\left(\tau \bar{d}_{1}^{2}+\cdots\right) / D_{2}\left(s_{1}, s_{2}\right)$

$M_{2}^{a b}\left(s_{1}, s_{2}\right)=\left(2 \tau \bar{d}_{1} \bar{v}_{g}+\cdots\right) / D_{2}\left(s_{1}, s_{2}\right)$

$D_{2}\left(s_{1}, s_{2}\right)=\left(2 \bar{v}_{o}-\bar{v}_{g}\right)+C R\left(\bar{v}_{o}-\bar{v}_{g}\right)\left(s_{1}+s_{2}\right)$

Similarly for $M_{3}^{a a a}, M_{3}^{b b b}, M_{3}^{a a b}$ and $M_{3}^{a b b}$ 
Since the Volterra transfer functions that differ only by the permutations of their arguments are lumped together to form the coefficient of a particular exponential, the first three symmetrized Volterra transfer functions can be expressed as

$H_{1}^{a}(s)=M_{1}^{a}(s)$

$H_{1}^{b}(s)=M_{1}^{b}(s)$

$H_{2}^{a a}\left(s_{1}, s_{2}\right)=M_{2}^{a a}\left(s_{1}, s_{2}\right) / 2$

$H_{2}^{b b}\left(s_{1}, s_{2}\right)=M_{2}^{b b}\left(s_{1}, s_{2}\right) / 2$

$H_{2}^{a b}\left(s_{1}, s_{2}\right)=M_{2}^{a b}\left(s_{1}, s_{2}\right)$

$H_{3}^{a a a}\left(s_{1}, s_{2}, s_{3}\right)=M_{3}^{a a a}\left(s_{1}, s_{2}, s_{3}\right) / 6$

$H_{3}^{b b b}\left(s_{1}, s_{2}, s_{3}\right)=M_{3}^{b b b}\left(s_{1}, s_{2}, s_{3}\right) / 6$

$H_{3}^{\alpha a b}\left(s_{1}, s_{2}, s_{3}\right)=M_{3}^{a a b}\left(s_{1}, s_{2}, s_{3}\right) / 2$

$H_{3}^{a b b}\left(s_{1}, s_{2}, s_{3}\right)=M_{3}^{a b b}\left(s_{1}, s_{2}, s_{3}\right) / 2$

which are used to construct the Volterra model of the boost converter operating in the discontinuous conduction mode.

\section{B. Buck Converter}

Similar to those derivations for the boost converter, the desired large-signal dynamical equation can be obtained as

$\dot{v}_{o}=\frac{d_{1}^{2} v_{g}\left(v_{g}-v_{o}\right) T_{s}}{2 C L v_{o}}-\frac{v_{o}}{C R}$

By perturbing (18) and then separating the steady-state and perturbed quantities, the following equations can be obtained which describe the Volterra model of the buck converter operating in the discontinuous conduction mode.

$$
\begin{aligned}
& \bar{v}_{o}=\frac{2 \bar{v}_{g}}{1+\sqrt{1+\frac{8}{\tau \bar{d}_{1}^{2}}}} \\
& M_{1}^{a}(s)=2 \tau \bar{d}_{1} \bar{v}_{g}\left(\bar{v}_{g}-\bar{v}_{o}\right) / D_{1}(s) \\
& M_{1}^{b}(s)=\tau \bar{d}_{1}^{2}\left(2 \bar{v}_{g}-\bar{v}_{o}\right) / D_{1}(s) \\
& D_{1}(s)=\left(\tau \bar{d}_{1}^{2} \bar{v}_{g}+4 \bar{v}_{o}\right)+2 C R \bar{v}_{o} s \\
& M_{2}^{a a}\left(s_{1}, s_{2}\right)=\left(2 \tau \bar{v}_{g}\left(\bar{v}_{g}-\bar{v}_{o}\right)-\cdots\right) / D_{2}\left(s_{1}, s_{2}\right) \\
& M_{2}^{b b}\left(s_{1}, s_{2}\right)=\left(2 \tau \bar{d}_{1}^{2}-\cdots\right) / D_{2}\left(s_{1}, s_{2}\right) \\
& M_{2}^{a b}\left(s_{1}, s_{2}\right)=\left(2 \tau \bar{d}_{1}\left(2 \bar{v}_{g}-\bar{v}_{o}\right)-\cdots\right) / D_{2}\left(s_{1}, s_{2}\right) \\
& D_{2}\left(s_{1}, s_{2}\right)=\left(\tau \bar{d}_{1}^{2} \bar{v}_{g}+4 \bar{v}_{o}\right)+2 C R \bar{v}_{o}\left(s_{1}+s_{2}\right)
\end{aligned}
$$

Similarly for $M_{3}^{a a a}, M_{3}^{b b b}, M_{3}^{a a b}$ and $M_{3}^{a b b}$

\section{Buck-Boost Converter}

Similarly, the corresponding equations for the buck-boost converter operating in the discontinuous conduction mode are given by $\dot{v}_{o}=\frac{d_{1}^{2} v_{g}^{2} T_{s}}{2 C L v_{o}}-\frac{\dot{v}_{o}}{C R}$

$\bar{v}_{o}=\bar{d}_{1} \bar{v}_{g} \sqrt{\frac{\tau}{2}}$

$M_{1}^{a}(s)=\tau \bar{d}_{1} \bar{v}_{g}^{2} / D_{1}(s)$

$M_{1}^{b}(s)=\tau \bar{d}_{1}^{2} \bar{v}_{g} / D_{1}(s)$

$D_{1}(s)=2 \bar{v}_{o}+C R \bar{v}_{o} s$

$M_{2}^{a a}\left(s_{1}, s_{2}\right)=\tau \bar{v}_{g}^{2} / D_{2}\left(s_{1}, s_{2}\right)-M_{1}^{a}\left(s_{1}\right) M_{1}^{a}\left(s_{2}\right) / \bar{v}_{o}$

$M_{2}^{b b}\left(s_{1}, s_{2}\right)=\tau \bar{d}_{1}^{2} / D_{2}\left(s_{1}, s_{2}\right)-M_{1}^{b}\left(s_{1}\right) M_{1}^{b}\left(s_{2}\right) / \bar{v}_{o}$

$M_{2}^{a b}\left(s_{1}, s_{2}\right)=2 \tau \bar{d}_{1} \bar{v}_{g} / D_{2}\left(s_{1}, s_{2}\right)-M_{1}^{a}\left(s_{1}\right) M_{1}^{b}\left(s_{2}\right) / \bar{v}_{o}$

$D_{2}\left(s_{1}, s_{2}\right)=2 \bar{v}_{o}+C R \bar{v}_{o}\left(s_{1}+s_{2}\right)$

Similarly for $M_{3}^{a a a}, M_{3}^{b b b}, M_{3}^{a a b}$ and $M_{3}^{a b b}$

\section{MODELING RESULTS}

In order to evaluate the converter nonlinear dynamical performance, both the duty ratio and source voltage excitations are incorporated with large-signal perturbations which are given by

$d_{1}=0.5+0.1 \cos (2 \pi 200 t)+0.05 \cos (2 \pi 300 t+\pi / 2)$

$v_{g}=20+4 \cos (2 \pi 600 t+\pi)+2 \cos (2 \pi 700 t+3 \pi / 2) \quad V$

The converter parameters are selected as $L=50 \mu \mathrm{H}$, $C=10 \mu \mathrm{F}, R=100 \Omega$ and $f_{s}=50 \mathrm{kHz}$ in such a way that the converter can operate in the discontinuous conduction mode. Moreover, another typical switching frequency $f_{s}=10 \mathrm{kHz}$ is adopted to investigate the vulnerability of the converter output response to different switching frequencies. In order to compare the dynamical performance among the boost, buck and buck-boost converters, their input excitations and circuit parameters are kept unchanged.

By using (9) and (12)-(17), the output voltage response of the boost converter due to the excitations given by (28) can be obtained. Spectral components, including both DC and AC terms, for two typical switching frequencies are listed in Table II. Furthermore, the spectral components are normalized by the corresponding DC term, hence the resulting spectra are shown in Fig. 4. Firstly, it can be found that both the spectral magnitudes and their normalized values depend on switching frequencies. Notice that they are frequency independent for all PWM converters operating in the continuous conduction mode. Secondly, it can be seen that the $\mathrm{DC}$ term, namely $0 \mathrm{~Hz}$, is not only contributed by the steady-state component $\bar{v}_{o}$ resulting from (9), but also by the components $\left(s_{a i}-s_{a i}\right)$ and $\left(s_{b i}-s_{b i}\right)$ which are the 2nd-order responses due to the intermodulation of the same tone. Thirdly, apart from the fundamental frequency components, namely $200 \mathrm{~Hz}, 300 \mathrm{~Hz}$, 
$600 \mathrm{~Hz}$ and $700 \mathrm{~Hz}$, due to the input excitations, there are significant subharmonic and higher harmonic frequency components. These additional frequencies are due to the converter nonlinearity.

Similarly, the output voltage response of the buck converter can be obtained by using (19)-(22) and (15)-(17). The resulting spectral magnitudes are also listed in Table II, and their normalized values are shown in Fig. 5. Similar to the boost converter, both the spectral magnitudes and their normalized values of the buck converter are frequency dependent. Additional DC offset as well as significant subharmonic and higher harmonic frequency components also exist.

For the buck-boost converter, its output voltage response can be obtained by using (24)-(27) and (15)-(17). The resulting spectral magnitudes are also listed in Table II. The spectral magnitudes are frequency dependent, and there are additional DC offset as well as significant subharmonic and higher harmonic frequency components. However, it should be noted that the normalized spectral values shown in Fig. 6 are independent of switching frequencies. This phenomenon can be explained by observing that all LHS variables in (24)-(27) are directly proportional to $\sqrt{\tau}$, and hence the normalized spectral components are independent of $T_{s}$ or $f_{s}$.

TABLE II

\section{OUTPUT VOLTAGE SPECTRA AT 50 AND $10 \mathrm{KHZ}$}

\begin{tabular}{lllllll}
\hline $\begin{array}{c}\text { Freq. } \\
(\mathrm{Hz})\end{array}$ & \multicolumn{2}{c}{$\begin{array}{c}\text { Boost } \\
(\mathrm{V})\end{array}$} & \multicolumn{2}{c}{$\begin{array}{c}\text { Buck } \\
(\mathrm{V})\end{array}$} & \multicolumn{2}{c}{$\begin{array}{c}\text { Buck-Boost } \\
(\mathrm{V})\end{array}$} \\
\hline & $50 \mathrm{k}$ & $10 \mathrm{k}$ & $50 \mathrm{k}$ & $10 \mathrm{k}$ & $50 \mathrm{k}$ & $10 \mathrm{k}$ \\
\hline $\bar{v}_{o}$ & 55.83 & 110.5 & 17.08 & 19.26 & 44.72 & 100.0 \\
0 & 56.89 & 112.3 & 17.12 & 19.30 & 45.35 & 101.4 \\
100 & 0.85 & 1.41 & 0.06 & 0.05 & 0.52 & 1.15 \\
200 & 7.79 & 17.23 & 0.92 & 0.30 & 7.53 & 16.83 \\
300 & 4.04 & 8.73 & 0.54 & 0.18 & 3.73 & 8.34 \\
400 & 1.23 & 2.57 & 0.36 & 0.15 & 1.06 & 2.36 \\
500 & 0.53 & 1.05 & 0.21 & 0.09 & 0.41 & 0.91 \\
600 & 6.09 & 10.98 & 2.98 & 3.78 & 4.13 & 9.24 \\
700 & 2.69 & 4.83 & 1.43 & 1.88 & 1.82 & 4.06 \\
800 & 0.35 & 0.85 & 0.21 & 0.11 & 0.38 & 0.84 \\
900 & 0.37 & 0.85 & 0.21 & 0.10 & 0.37 & 0.83 \\
1000 & 0.14 & 0.27 & 0.06 & 0.04 & 0.11 & 0.24 \\
1100 & 0.05 & 0.07 & 0.02 & 0.02 & 0.02 & 0.04 \\
1200 & 0.21 & 0.29 & 0.05 & 0.01 & 0.09 & 0.19 \\
1300 & 0.22 & 0.30 & 0.04 & 0.01 & 0.09 & 0.19 \\
1400 & 0.07 & 0.09 & 0.01 & 0.00 & 0.02 & 0.05 \\
1500 & 0.03 & 0.04 & 0.00 & 0.00 & 0.01 & 0.03 \\
1600 & 0.02 & 0.02 & 0.00 & 0.00 & 0.01 & 0.01 \\
1700 & 0.00 & 0.00 & 0.00 & 0.00 & 0.00 & 0.00 \\
1800 & 0.02 & 0.02 & 0.00 & 0.00 & 0.00 & 0.01 \\
1900 & 0.02 & 0.02 & 0.01 & 0.00 & 0.01 & 0.01 \\
2000 & 0.01 & 0.01 & 0.00 & 0.00 & 0.00 & 0.01 \\
2100 & 0.00 & 0.00 & 0.00 & 0.00 & 0.00 & 0.00 \\
\hline & & & & & &
\end{tabular}

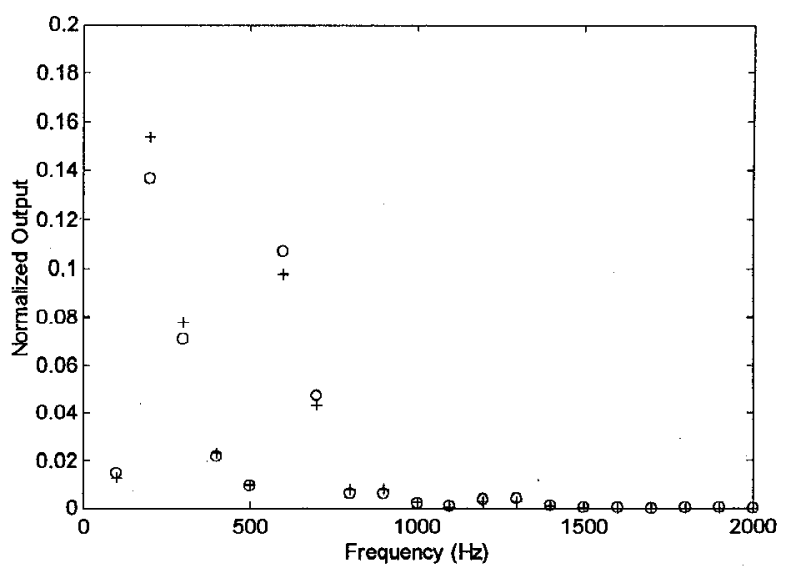

Fig. 4 Boost converter spectra at different switching frequencies: ' $\mathrm{O}$ ' $50 \mathrm{kHz}$; '+' $10 \mathrm{kHz}$

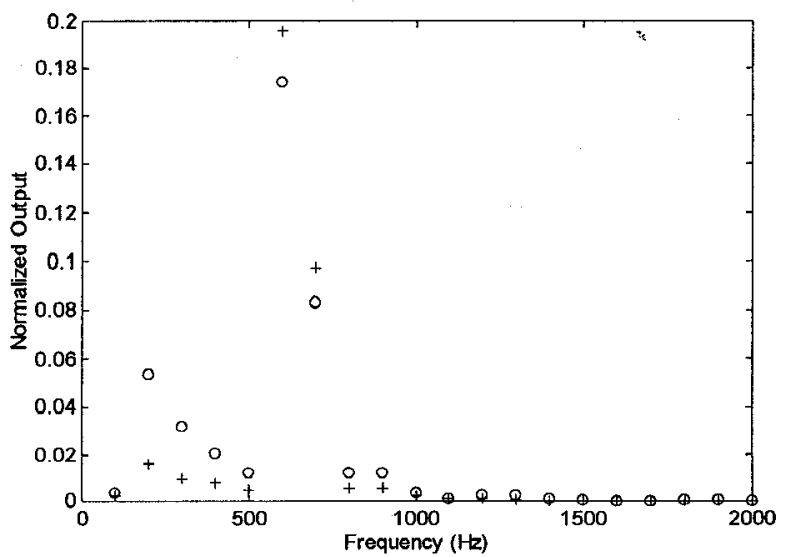

Fig. 5 Buck converter spectra at different switching frequencies: ' $\mathrm{O}$ ' $50 \mathrm{kHz}$; '+' $10 \mathrm{kHz}$

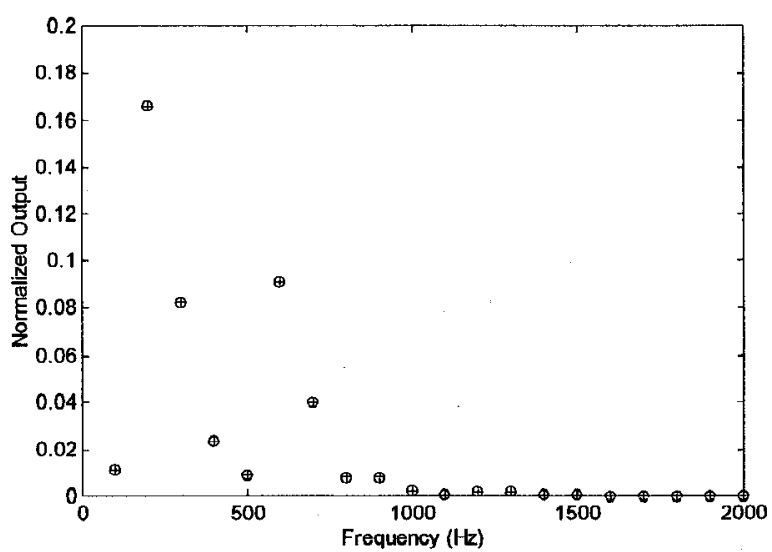

Fig. 6 Buck-boost converter spectra at different switching frequencies: ' $\mathrm{O}$ ' $50 \mathrm{kHz}$; '+' $10 \mathrm{kHz}$ 


\section{VERIFICATION}

To verify the proposed approach, the modeling results are compared with the simulated results obtained by PSpice simulation. The experimental verification is not carried out because the modeling accuracy can hardly be proved in the presence of inevitable experimental errors. The PSpice simulation involves a tedious transient analysis from start-up to steady-state and a Fourier analysis of the resulting transient waveform over a defined period.

Based on the aforementioned input excitations and circuit parameters, the PSpice-simulated output voltage and inductor current waveforms of the boost converter operating at $50 \mathrm{kHz}$ are shown in Fig.7(a). The inductor current waveform verifies that the converter operates in the discontinuous conduction mode. The corresponding output voltage spectrum obtained by PSpice simulation is marked ' $x$ ' and is compared with the modeling results marked ' $\mathrm{O}$ ' as shown in Fig. 7(b). It is obvious that the agreement between the results is very good.

Similarly, the PSpice-simulated inductor current waveforms of the buck and buck-boost converters operating at $50 \mathrm{kHz}$ can verify their discontinuous conduction mode of operation as well as the accuracy and applicability of the modeling approach. It should be noted that the PSpicesimulated results are obtained at the expense of an hour while the modeling results can be obtained in less than a second. Moreover, numerical simulation can never provide an analytical model as given by the proposed approach.

\section{CONCLUSIONS}

An analytical spectral modeling approach for switchedmode PWM converters, operating in the discontinuous conduction mode, has been presented. The modeling approach has been successfully applied to various PWM converters, including the boost, buck and buck-boost, to investigate their spectral responses due to large-signal perturbations of both the duty ratio and source voltage. In the output voltage spectra of these converters, it can be found that there are additional DC offset as well as significant subharmonic and higher harmonic frequency components, which are omitted in small-signal analysis. Contrary to the frequency independence in the continuous conduction mode, their spectral magnitudes for the discontinuous conduction mode of operation are influenced by switching frequencies. Moreover, it is interested to note that the normalized spectral values of the buck-boost converter are independent of switching frequencies while those of the boost and buck converters are frequency dependent. The modeling results has been compared with the PSpice-simulated results, and hence the modeling accuracy and applicability are verified. The approach is so general that it can readily be extended to other switched-mode power converters.

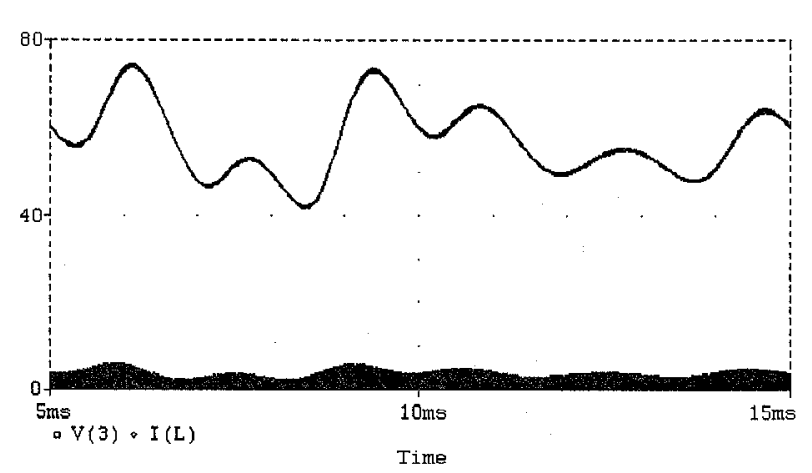

(a)

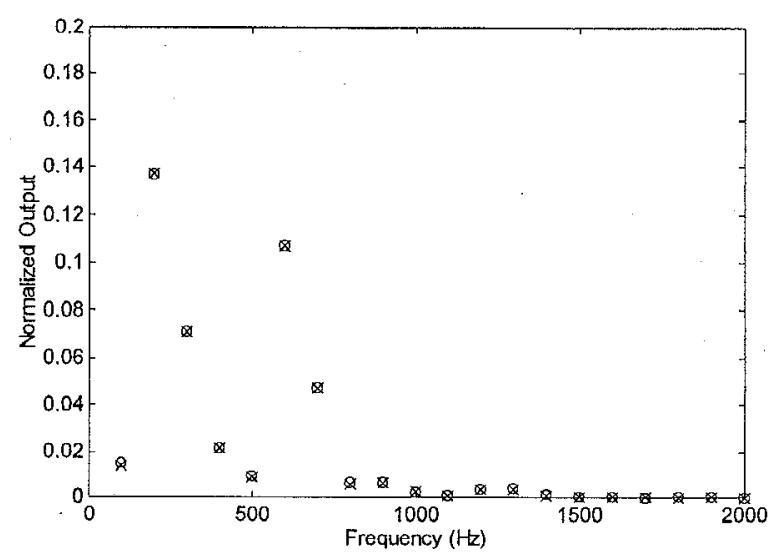

(b)

Fig. 7 Verification of boost converter: (a) PSpice-simulated output voltage (upper trace) and inductor current (lower trace) waveforms (b) normalized output voltage spectra with ' $O$ ' resulting from modelling and ' $x$ ' from PSpice simulation

\section{REFERENCES}

[1] R. Tymerski, "Volterra series modeling of power conversion systems," IEEE Trans. Power Electronics, Vol. 6, 1991, pp. 712-718.

[2] C.C. Chan and K.T. Chau, "Spectral Modeling of switched-mode power converters," IEEE Trans. Industrial Electronics, Vol. 41, 1994, pp. 441-450.

[3] S. Cuk, and R.D. Middlebrook, "A general unified approach to modeling switching dc-to-dc converters in discontinuous conduction mode," in Proc. IEEE Power Electronics Specialists' Conference, 1977, pp. 36-57.

[4] E. Bedrosian, and S.O. Rice, "The output properties of Volterra systems (nonlinear systems with memory) driven by harmonic and Gaussian inputs," Proceedings of IEEE, Vol. 59, 1971, pp. 1688-1707. 